\title{
Efeitos de Espécies de Timbós (Derris spp.: Fabaceae) em Populações de Musca domestica $\mathbf{L}$.
}

\author{
José P. C. da Costa ${ }^{1}$, Muracy Belo ${ }^{2,4}$ e José C. Barbosa ${ }^{3}$ \\ 'Área Técnica de Recursos Genéticos e Biotecnologia, EMBRAPA-CPATU, Caixa postal \\ 48, 66095-100, Belém, PA. \\ ${ }^{2}$ Departamento de Biologia Aplicada à Agropecuária, FCAV-UNESP, 14870-000, \\ Jaboticabal, SP. \\ ${ }^{3}$ Departamento de Ciências Exatas, FCAV-UNESP, 14870-000, Jaboticabal, SP. \\ ${ }^{4}$ Autor correspondente.
}

An. Soc. Entomol. Brasil 26(1): 163-168 (1997)

Effects of Timbó (Derris spp.: Fabaceae) Species $m$ Musca domestica L. Populations

\begin{abstract}
The effect of the root powder of two timbó species (Derris urucu and D. nicou) on Musca domestica L. larvae was estimated for populations of two strains of this fly collected from two localities of São Paulo state, i.e., Jaboticabal (Jab) and Brodósqui (Bro). Analysis was performed using a logistic model, contrasting the values for the regression equations of lethal doses of the two timbó species for both fly strains. Data showed that D. urucu was more efficient than D. nicou to control the two strains, and more than twice the amount of D. nicou powder was necessary to get the same effect caused by D. urucu. There was specificity in the action of the timbó species on the fly strains. $D$. иrucu was more efficient to control of Bro strain, while D. nicou presented more control on Jab strain.
\end{abstract}

KEY WORDS: Insecta, flies, rotenone, control.

RESUMO - A partir de ensaios com dosagens crescentes, foi avaliado o efeito do pó da raiz de duas espécies de timbó (Derris urucu e D. nicou) sobre populações de larvas de duas linhagens de Musca domestica L., provenientes de duas localidades do Estado de São Paulo, Jaboticabal (Jab) e Brodósqui (Bro). Para obtenção das doses letais foram utilizados ajustes de regressão de acordo com o modelo logístico. D. urucu foi mais eficiente que $D$. nicou no controle das duas linhagens, sendo necessário mais que o dobro da quantidade de D. nicou para se obter os mesmos efeitos causados com D. urucu. Foi demonstrada a existência de especificidade de ação dos timbós nas linhagens de moscas. D, игиси foi mais eficiente no controle da linhagem Bro, enquanto que D. nicou controlou maior número de indivíduos da linhagem Jab.

PALAVRAS-CHAVE: Insecta, moscas, rotenona, controle.

Os timbós, devido a sua toxicidade, são plantas utilizadas pêlos índios da Amazônia nas pescarias. As espécies de maior importân- cia são Derris urucu e D. nicou. Lima (1987), relata que substâncias encontradas nas raízes, como a rotenona e os rotenóides, entre os 
quais se destacam a deguelina, tefrosina e o toxicarol, são produtos de onde deriva a importância destas plantas.

Segundo Caminha Filho (1940) antes de 1946, a rotenona era utilizada como inseticida nas lavouras contra insetos (larvas de borboletas, coccídeos, cochonilhas e pulgões) e ectoparasitas de animais. Costa et al. (1986), relata o sucesso de D. urucu (usado como solução do pó da raiz) no controle ao piolho (Haematopinus tuberculatus) dos búfalos. A despeito do conhecimento da rotenona ser inócua a animais de sangue quente (Lima 1987), ela é tóxica para mamíferos (Link 1965), sendo absorvida através da pele, porém, relativamente inofensiva quando utilizada adequadamente. Estes fatos enfatizam a possibilidade dessas plantas serem empregadas em fazendas no controle de Musca domestica L. Desta forma, testou-se o efeito tóxico de $D$. urucu e D. nicou para larvas dessa espécie.

\section{Material e Métodos}

As linhagens de moscas empregadas foram coletadas com rede entomológica (confeccionada com tecido de "organza" branco) em granjas de galinhas poedeiras, localizadas nos municípios de Brodósqui-SP (linhagem Bro) e Jaboticabal-SP (linhagem Jab). Segundo Oliveira et al. (1 993), estas linhagens diferem em relação à resistência para três tipos de inseticidas e no desempenho apresentado na exploração dos recursos disponíveis em laboratório, apesar de serem coletadas em localidades próximas (cerca de $70 \mathrm{Km}$ de distância).

Todo procedimento posterior foi realizado dentro de câmara climatizada $\left(27 \pm 2^{\circ} \mathrm{C}, 65\right.$ $70 \%$ U. R. e fotoperíodo de 12 horas). As moscas capturadas foram mantidas neste local, para o fornecimento de ovos e destes foram obtidas larvas com dois dias de idade. As populações experimentais foram montadas com 300 dessas larvas, em recipientes de plástico transparentes $(500 \mathrm{ml})$ e com meio de cultura $(31,4 \mathrm{~g}$ de farelo de trigo, 2,4 $\mathrm{g}$ de fermento comercial, $1,5 \mathrm{~g}$ de leite em pó integral e $60 \mathrm{ml}$ de água destilada). Na tampa do recipiente foi feita uma abertura de $3 \times 3 \mathrm{~cm}$ vedada com tecido de "organza" para permitir a aeração e evitar a saída das larvas e imagos.

As espécies de timbó $D$. иrucu e D. nicou, (clones $n^{\circ} 52$ e 498) foram coletadas respectivamente nos municípios de São Paulo de Olivença-AM e Calçoene-AP. As raízes dos timbós foram adquiridas do Banco Ativo de Germoplasma (BAG) da EMBRAPA-CPATU em Belém-PA e submetidas a secagem em estufa com ventilação forçada, em temperatura de $40 \pm 2{ }^{\circ} \mathrm{C}$. Em seguida, foram trituradas separadamente em moinho tipo Willey (Tecnal), modelo TB-340, com uso de uma peneira de 40 mesh, para formação do pó de timbó.

Os experimentos (quatro ensaios com três repetições), foram iniciados com populações de larvas em meio de cultura, no qual a quantidade de farelo de trigo foi substituída proporcionalmente pelas quantidades de pó de timbó acrescidas. Os dois primeiros ensaios envolveram os clones de $D$. urucu e $D$. nicou e a linhagem Jab, com oito tratamentos: 0,0 (controle), 0,5, 1,0, 1,5, 2,0, 3,0, 4,0 e 5,0 g. Os dois últimos ensaios foram realizados com os clones de $D$. urucu e $D$. nicou e a linhagem Bro, com os tratamentos: 0,0 (controle), 0,5, 1,0, 1,5 e $2,0 \mathrm{~g}$.

As avaliações do número de imagos emergidos foram feitas diariamente, e as análises foram realizadas através do modelo logístico (Finney 1971), o que permitiu determinar os valores das doses letais para cada linhagem de moscas (foi empregado o teste t para avaliar a significância dos parâmetros a e b do modelo), bem como, a comparação entre as curvas obtidas para cada espécie de timbó (foi empregado o teste de quiquadrado para avaliar a significância das condições de paralelismo e coincidência das linhas de regressão), em cada linhagem de mosca.

\section{Resultados}

Os resultados mostraram que $D$. иrucu foi mais eficiente no controle das moscas das duas 
linhagens, pois com o tratamento de $0,7 \mathrm{~g}$ de pó da raiz, as populações Jab e Bro atingiram percentagem de mortalidade maior que $80 \%$ (Fig. 1), enquanto que o mesmo tratamento com D. nicou controlou cerca de $10 \%$ da linhagem Jab. Nas populações de moscas da linhagem
Bro não ocorreram reações negativas aparentes no número de moscas emergidas para tal dosagem. Os dados indicaram que no controle das moscas houve especificidade de ação entre as espécies de Derris e as linhagens de M. domestica. Deste modo, a linhagem Bro
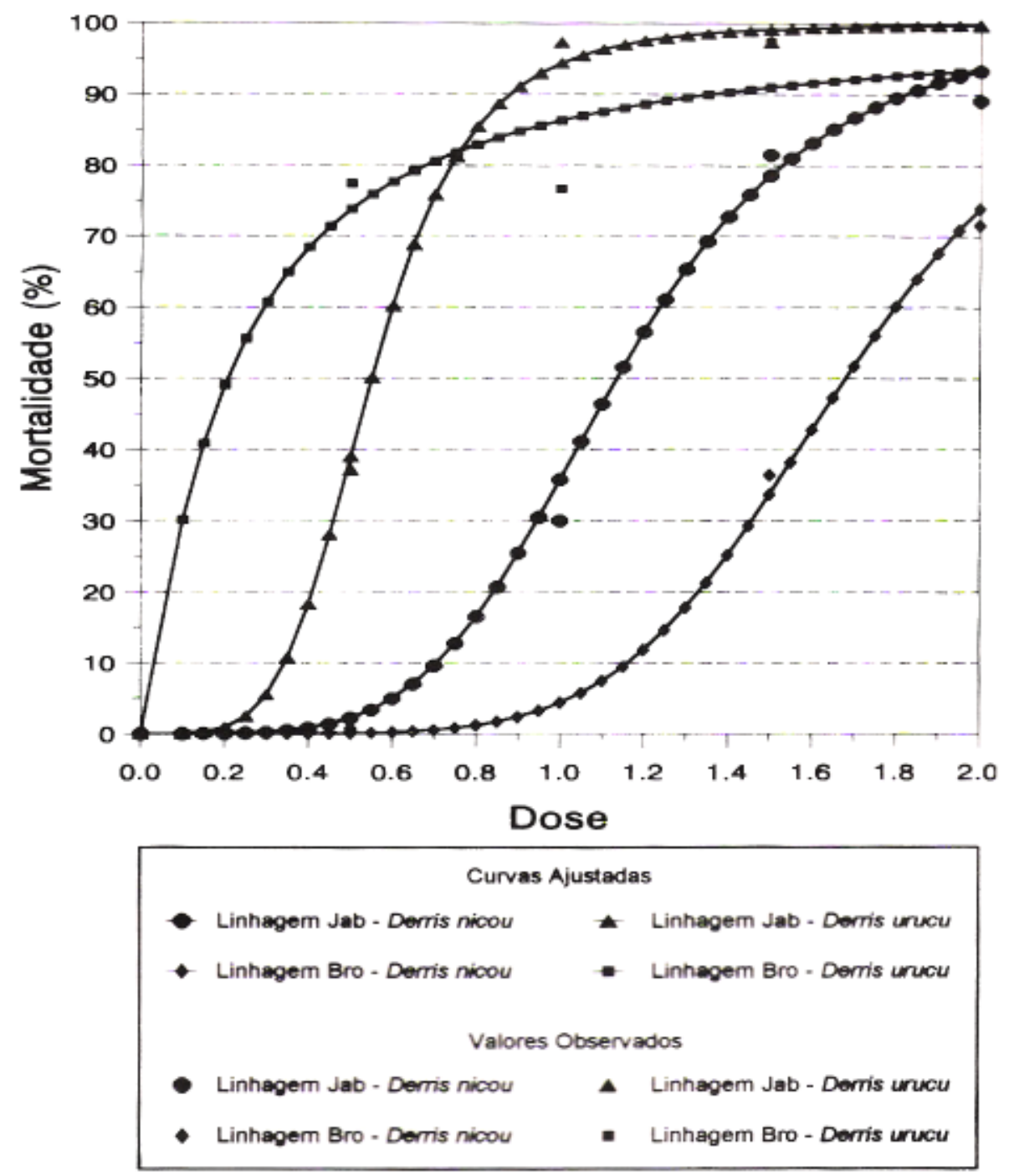

Figura 1. Mortalidade (\%) observadas (descontando-se a mortalidade natural) e estimadas através das equações de regressão para duas linhagens de Musca domestica (Jab - Jaboticabal, SP e Bro - Brodósqui, SP) em populações tratadas com diferentes doses do pó de timbó, Derris nicou e Derris urucu 
Tabela 1 - Equações obtidas a partir de bioensaios e ajustadas de acordo com o modelo logístico e dose letal $\left(\mathrm{DL}_{50}\right)$ do pó da raiz de duas espécies de timbó - Derris nicou e Derris игиси, sobre duas linhagens de Musca domestica - Jab (Jaboticabal,SP) e Bro (Brodósqui,SP).

\begin{tabular}{|c|c|c|c|c|}
\hline Espécie & Equação Ajustada & Tes & & \\
\hline de timbó & $\mathrm{Li}=\mathrm{a}+\mathrm{b} \log _{10} \mathrm{Di}$ & a & b & \\
\hline Derris nicou - Jab & $\mathrm{Li}=-0,5852+10,6940 \log _{10} \mathrm{Di}$ & $6,63 * *$ & $22,31 * *$ & 1,1343 \\
\hline Derris nicou - Bro & $\mathrm{Li}=-3,0968+13,7399 \log _{10} \mathrm{Di}$ & $14,38 * *$ & $16,34 * *$ & 1,6803 \\
\hline Derris urucu - Jab & $\mathrm{Li}=2,8200+10,8476 \log _{10} \mathrm{Di}$ & $20,12 * *$ & $20,33 * *$ & 0,5496 \\
\hline Derris urucu - Bro & $\mathrm{Li}=1,8392+2,6829 \log _{10} \mathrm{Di}$ & $28,86 * *$ & $10,57 * *$ & 0,2063 \\
\hline
\end{tabular}

** significativo ao nível de $1 \%$ de probabilidade.

foi mais sensível que a linhagem Jab ao $D$. итиси, enquanto que os indivíduos da linhagem Jab mostraram maior sensibilidade do que a linhagem Bro a D. nicou.

As comparações dos efeitos das espécies de timbós nas populações de moscas indicaram que é necessária maior quantidade de $D$. nicou para se obter a DL para as moscas da linhagem Bro (1,68 g), em relação à linhagem $\mathrm{Jab}(1,13 \mathrm{~g})$, enquanto que, maior quantidade de D. иrucu é necessária para obter a $\mathrm{DL}_{50}$ na linhagem $\mathrm{Jab}(0,55 \mathrm{~g})$, em relação à linhagem Bro (0,21 g) (Tabela 1).

Ocorreram diferenças significativas entre as linhas de regressão obtidas para cada espécie de timbó em cada linhagem de mosca

Tabela 2 - Valores obtidos para o teste $\chi^{2}$ de paralelismo e coincidência para as comparações entre as equações de regressão obtidas para a taxa de mortalidade de duas linhagens de moscas (Jab - Jaboticabal,SP e Bro - Brodósqui,SP), expostas a concentrações crescentes $(0,5 ; 1,0 ; 1,5$ e 2,0 g) de pó da raiz de Derris nicou e Derris urucu.

\begin{tabular}{|c|c|c|c|c|c|c|c|}
\hline \multirow{2}{*}{$\begin{array}{l}\text { Espécie } \\
\text { de timbó }\end{array}$} & \multicolumn{2}{|c|}{ Linha- } & \multirow{2}{*}{$\begin{array}{l}\text { Espécie } \\
\text { de timbó }\end{array}$} & \multirow{2}{*}{$\begin{array}{l}\text { Linha- } \\
\text { gem }\end{array}$} & \multicolumn{2}{|c|}{ Teste do Qui-quadrado } & \multirow{2}{*}{ Potência } \\
\hline & gem & & & & Paralelismo & Coincidência & \\
\hline D. nicou & $\mathrm{Jab}$ & vs. & D. nicou & Bro & $11,35^{* *}$ & $485,57 * *$ & 0,6830 \\
\hline D. urucu & $\mathrm{Jab}$ & vs. & D. urucu & Bro & 272,47 ** & $276,70 * *$ & 1,0796 \\
\hline D. nicou & $\mathrm{Jab}$ & vS. & D. игиси & $\mathrm{Jab}$ & $0,05^{\mathrm{NS}}$ & $915,89 * *$ & 2,0675 \\
\hline D. nicou & Bro & vs. & D. urucu & Bro & $276,81 * *$ & $2133,10 * *$ & 4,7018 \\
\hline
\end{tabular}

NS = não significativo ao nível de $5 \%$ de probabilidade.

** = significativo ao nível de $1 \%$ de probabilidade. 
(Tabela 2). Verifica-se que os testes para paralelismo e coincidência das linhas de regressão foram significativos para todas as comparações, com exceção do teste de paralelismo para a linhagem Jab em D. nicou contra a linhagem Jab em D. игисu, indicando que as espécies de timbós, possuem eficiências diferentes no controle das larvas de moscas das duas linhagens estudadas.

A linhagem Jab foi significativamente mais afetada por D. nicou que a linhagem Bro. Quanto a $D$. urucu, verifica-se o contrário. As duas comparações mostraram que, quantitativamente, são necessárias duas vezes mais $D$. nicou que $D$. urucu, para controlar o mesmo número de indivíduos nas populações da linhagem Jab. Esta quantidade, em relação à linhagem Bro, representa quase cinco vezes mais pó de $D$. nicou que devem ser empregados para controlar o mesmo número de indivíduos que D. urucu (Tabela 2).

\section{Discussão}

Segundo Lima (1947), existem diferentes características apresentadas por D. urucu $e$ D. nicou quando cultivadas e com as quais estas espécies podem ser diferenciadas. A primeira espécie é mais rústica, apresentando florescimento e frutificação; enquanto a segunda não apresenta florescimento, mesmo quando tratada com fito-hormônios, ou quando tem o prolongamento do fotoperíodo com iluminação artificial.

No D. urucu, os ramos tomam-se escandentes, entrelaçam-se formando um teto compacto sobre o solo, protegendo-o da ação do sol, além das raízes apresentarem nodosidade resultantes da simbiose com a bactéria Rhyzohium; já.D. nicou conserva seus ramos eretos. Destaca-se, porém, que $D$. urucu produz maior quantidade de raízes que $D$. nicou, além de apresentar a possibilidade de ser melhorado geneticamente.

Diversos autores, (Corbett 1940, Caminha Filho 1940, Lima 1947), citam D. nicou como mais rico em teor de rotenona que $D$. иrисu.
De acordo com Corbett (1940) e Caminha Filho (1940), a quantidade de rotenona em $D$. nicou está entre 15 a $17 \%$ e, em D. urucu, entre 5 a $12 \%$. Apesar das citações acima sobre o teor de rotenona, os dados mostraram ação mais eficiente de $D$. иrucu no controle dos indivíduos das duas linhagens de $M$. domestica. Mostrando a possibilidade de outras substâncias além da rotenona, como aquelas mais conhecidas, tais como deguelina, tefrosina e toxicarol (Lima 1947), além de outras desconhecidas, como a nilobilina, um alcalóide que, de acordo com Caminha Filho (1 940), foi encontrada por Groffoy em 1895, e que poderiam estar agindo no controle das larvas. Estes fatos indicam a necessidade de mais pesquisas para se detectar outros produtos que poderiam estar agindo como princípio ativo destas plantas.

Por outro lado, a despeito do efeito marcante de $D$. urucu, em relação à $D$. nicou, no controle das populações de $M$. domestica, pode-se destacar que a linhagem Jab foi mais sensível que a Bro em relação à $D$. nicou. Quanto à $D$. urucu, verificou-se o contrário, isto é, a linhagem Bro foi mais sensível. Estes fatos destacam a especificidade de ação do pó de timbó nas linhagens de $M$. domestica. Assim, os dados mostram a possibilidade de que com baixo custo, nas propriedades rurais, o pó de timbó pode ser obtido e usado no controle das larvas de um dos mais importantes vetores de endemias rurais.

\section{Agradecimentos}

Ao Prof. Dr. Dilermando Perecin, docente do Departamento de Ciências Exatas da FCAVUNESP, pela revisão e sugestões apresentadas.

\section{Literatura Citada}

Caminha Filho, A. 1940. Timbó e rotenona. Uma riqueza nacional inexplorada. Rio de Janeiro, Serviço de Informação Agrícola, $14 \mathrm{p}$. 
Corbett, C. E. 1940. Plantas ictiotóxicas farmacologia da rotenona. São Paulo, Fac. Med. Univ. São Paulo, 157 p.

Costa, N. A. da, C. N. B. do Nascimento, L. O. D. de Moura Carvalho, S. Dutra, \& E. S. Pimentel, 1986. Uso do timbó urucu (Derris urucu) no controle do piolho (Haematopinus tuberculatus) em bubalinos. Belém, EMBRAPA-CPATU, Bol. Pesq, 78,16 p.

Finney, D. J. 1971. Probit analysis. 3rd ed., Cambridge, Cambridge University Press, 333p.

Lima, R. R. 1947. Os timbós da Amazônia brasileira. Rio de Janeiro, Ministério da Agricultura, Bol. Tec. 36, p. 14-29.
Lima, R. R. 1987. Informações sobre duas espécies de timbó Derris urucu (Killip et Smith) MacBride e Derris nicou (Killip et Smith) MacBride, como plantas inseticidas. Belém, EMBRAPA-CPATU, Documentos, 42, $23 \mathrm{p}$.

Link, R. P. 1965. Insecticides. p.702-726. In: L. M. Jones, (ed.) Veterinary pharma-cology and therapeutics. 3rd ed., Ames, Iowa State University.

Oliveira, P. C. S., L. K. G. Trevijano, \& M. Belo. 1993. Sensibilidade estacional em linhagens de Musca domestica (L.) para três tipos de inseticidas. An. Soc. Entomol. Brasil 22:455-461.

Recebido em 25/09/95. Aceito em 30/02/97. 